\title{
Exteraction and Characterization Some Fatty Acid of the Seeds Oil from Albizialebbeck (L.) Benth
}

Fath El-Rahman Ahmed ${ }^{1 *}$, Munged I ${ }^{2}$, Mohammed Algozoly ${ }^{1}$, Saad Badwi ${ }^{1}$, Awd Hamad ${ }^{1}$, Abdel Fathah B $^{1}$, Masri Abdel Moneam ${ }^{1}$

${ }^{1}$ Department of Chemistry \& Biology, Faculty of Education, Omdurman Islamic University, Khartoum, Sudan

${ }^{2}$ Department of Chemistry, Faculty of Applied \& Industrial Sciences, Bahri University, Khartoum, Sudan

*Corresponding author: Fath El-Rahman Ahmed

Abstract

Albizialebbeck(L.) Benth. A member of Fabaceae family, have numerous medicinal and pharmacological properties, the plant was collected from Elfitahab areas in Omdurman city. The fruit pulp of Albizialebbeck(L.) Benth. was subjected to continuous and successive soxhlet extraction with n-Hexane. The oil extract was screened with antimicrobial activity and analysis by GC/MS technique to appeared 19 components.

Keywords: Albizialebbeck(L.) Benth, GC/MS, Antimicrobial activity, Soxhlet.

Copyright @ 2020: This is an open-access article distributed under the terms of the Creative Commons Attribution license which permits unrestricted use, distribution, and reproduction in any medium for non-commercial use (NonCommercial, or CC-BY-NC) provided the original author and source are credited.

\section{INTRODUCTION}

Albizialebbeck(L.) Benth tree it is belonging to the family Fabaceae member of the subfamily Mimosae It is widely available plant in the tropical and subtropical Asia and Africa [1]. It is cultivated in many parts of Sudan as shade tree in front of houses, farmlands, along roadsides, along rivers, and as an ornamental plant in gardens due to its pleasant appearance. It evergreen tree with height about $18-30 \mathrm{~m}$ with a trunk $50 \mathrm{~cm}$ to $1 \mathrm{~m}$ in diameter and the bark is gray colour [2] with glabrous young shoots [3]. Leaves are evenly bi pinnate and the leaflets are in 5-9 pairs, 2.5-5.0 cm long, broadly oblong and pale green [1] flowers are to greenish-yellow ,fragarant in globose umbellate heads $2-3.8 \mathrm{~cm}$ diameter, stamens are longer than the corolla [2]. Pods are 10-30 cm long, 2-4.5 cm wide, thin, linear oblong, smooth, green turning yellowbrown on maturity, They have 4-12 yellowish brown seeds $[4,5]$. The plant have numerous medicinal and pharmacological properties, different parts of this plant have lots of useful properties, the roots are used in ophthalmia, alleviate spasms, rat bite, stimulate the cardiovascular system, hemicranias, anticancer and spermicidal properties, antifungal activity [2, 3] the bark is used for treatments of skin disease, relives toothache, hemorroid, leprosy, piles, deafness, cooling, alexiteric, pimples, itching, anthelmintic, syphilis, cures diseases of blood, leucoderma, itching, paralysis, cures diseases of blood, leucoderma, itching and body weakness, bubbles, boils, scabies, Inflammation, antidiabetic, bronchitis, over the top sweat, excessive perspiration, heaps, After drying and pounding, it is used as a soap substitute $[3,6,7,2]$. The leaves of this tree are good for syphilis, ophthalmic diseases, night blindness, nootropic activity, syphilis and ulcer, anxiolytic activity, cold, cough, respiratory disorder [3, $2]$. The leaves are also used as cattle fodder, mulch, and excrement because of high nitrogen contents $[3,6]$ Leaves are also used as soap substitute after being dried [7]. The flowers are used in treatment of opthalmia, aphrodisiac, emollient, night blindness, maturant, psychological disorders, spermatorrhea. insomnia and warts, Flowers also are applied as cataplasm on furuncles and used for retention of seminal fluid [3, 8].

The pods extract is reckoned have antidiabetic, antiprotozoal, antidiabetic, and anticancer properties and also show antispermatogenic effect [2, 3] and possesses a wide antibacterial property against E. coli and antiamoebic activity against Entamoeba histolytic [9]. The Seeds have been used for a variety of therapeutic purposes such as cure piles, can treat diabetes, anti-tumor, diarrhea, scrofulous swelling, aphrodisiac, brain tonic, gonorrhea and tuberculous glands [2, 10]. And have constipating properties, astringent. Are also used for treating hemorrhoid, it is believed that the extract of seed covers have anti protozoal properties [7], The seeds oil is applied topically to cure leucoderma [3]. The oliage is commonly used as fodder in India for cattle, and in Sudan, goats eat fallen leaves and flowers [11]. 


\section{MATERIALS AND METHODS}

Plant Material

Albizialebbeck(L.) Benth seeds were collected from faculty of agriculture Omdurman Islamic University Elfetehab area Omdurman city, Khartoum State (Sudan), during April 2018.The plant is authenticated by the Department of Biology and Chemistry faculty of Education, Omdurman Islamic University. The seeds of Albizialebbeck(L.) Benth were manually separated from the fruits, dried at room temperature at 8 days, $500 \mathrm{~g}$ were ground by an electric mixer until a fine powder is obtained $367 \mathrm{~g}$ and stored at room temperature until further use.

\section{Test Organisms}

Albizialebbeck(L.) Benth oil was screened for antibacterial and antifungal activities using the standard microorganisms shown in Table-1.

Table-1: Test organisms

\begin{tabular}{|l|l|l|l|}
\hline Ser. No & Micro organism & Abb. & Type \\
\hline 1 & Bacillus subtilis & B.s & G+ve \\
\hline 2 & Staphylococcus aureus & S.a & G+ve \\
\hline 3 & Pseudomonas aeruginosa & P.a & G-ve \\
\hline 4 & Escherichia coli & E.c & G-ve \\
\hline 5 & Candida albicans & C.a & Fungus \\
\hline
\end{tabular}

\section{Extraction of oil}

Powdered shade-dried seeds of Albizialebbeck(L.) Benth $\mathrm{s}(500 \mathrm{~g})$ were exhaustively extracted with n-hexane at room temperature. The solvent was removed in vauo and the oil was kept in the fridge at $4 \mathrm{oC}$ for further manipulation.

\section{Esterification of oil}

A Methanolic solution of sodium hydroxide was prepared by dissolving $(2 \mathrm{~g})$ of sodium hydroxide in $100 \mathrm{ml}$ methanol. A stock solution of methanolic sulphuric acid was prepared by mixing (1ml) of concentrated sulphuric acid with $(99 \mathrm{ml})$ methanol.

The oil (2ml) was placed in a test tube and $7 \mathrm{ml}$ of alcoholic sodium hydroxide were added followed by $7 \mathrm{ml}$ of alcoholic sulphuric acid. The tube was stoppered and shaked vigorously for five minutes and then left overnight. $(2 \mathrm{ml})$ of supersaturated sodium chloride were added, then $(2 \mathrm{ml})$ of normal hexane were added and the tube was vigorously shaked for five minutes. The hexane layer was then separated. $(5 \mu l)$ of the hexane extract were mixed with $(5 \mathrm{ml})$ diethyl ether. The solution was filtered and the filtrate $(1 \mu \mathrm{l})$ was injected in the GC-MS vial.

\section{Instruments}

Gas Chromatography coupled with Mass Spectrometry Analysis was conducted on a Shimadzo GC-MS-QP2010 Ultra instrument equipped with a splitsplitless injector and with a RTX-5MS column $(30 \mathrm{~m}$, length; $0.25 \mathrm{~mm}$ diameter; $0.25 \mu \mathrm{m}$, thickness). The GC oven was set to a temperature range of 150 to $3000 \mathrm{C}$ with $60 \mathrm{C} / \mathrm{min}$, and a solvent delay of $7 \mathrm{~min}$. The injector temperature was maintained at $300{ }^{0} \mathrm{C}$. The carrier gas of the sample was helium at $1.0 \mathrm{~mL} / \mathrm{min}$ and the sample was injected in the splitless mode. The MS conditions were the followings: ionization energy, 70 $\mathrm{eV}$; electronic impact ion source temperature, $200^{\circ} \mathrm{C}$; interface temperature, $25 \backslash 10^{\circ} \mathrm{C}$; scan rate $1.6 \mathrm{scan} / \mathrm{s}$; mass, 40-600 amu. For the identification the compounds the mass spectra of the samples were compared with determined by the chromatographic method with the help of NIST and WILEY library.

\section{Antimicrobial Assay}

One gram of the oil was weighed and dissolved in $10 \mathrm{ml}$ of DMSO to obtain a concentration of $100 \mathrm{mg} / \mathrm{ml}$. This was the initial concentration of the oil used to check the antimicrobial activities. Diffusion method was the method used for screening the oil. Mueller Hinton and Sabouraud dextrose agars were the media used as the growth media for the bacteria and the fungus respectively. The media were prepared according to the manufacturer's instructions, sterilized at $121 \mathrm{oC}$ for 15 minutes, poured into sterile Petri dishes and were allowed to cool and solidify. The sterilized media were sealed with $0.1 \mathrm{ml}$ of the standard inoculums of the test microbe (Mueller Hinton agar was sealed with the bacteria and Sabouraud dextrose agar sealed with the fungus). The inoculums were spread over the surface of the medium by the use of a sterile swab. By the use of a standard cork borer of $6 \mathrm{~mm}$ in diameters, a well was cut at the centre of each inoculated medium. Serial dilutions of the oil $(0.1 \mathrm{ml})$ were then introduced into the well on the inoculated medium. Incubation of the inoculated medium was made at $37 \mathrm{oC}$ for 24 hours for the bacteria and at $30 \mathrm{oC}$ and for 4 days for the fungus. After incubation each plate of the medium was observed for the growth inhibition zone. The zone was measured with a transparent ruler and the results were recorded in millimeters.

\section{RESULTS AND DISCUSSION Constituents of oil}

The GC-MS spectrum of the n-hexane extract of oil is shown in the Figure 1. Total of 19 compounds present in the $n$-hexane extract were determined by the chromatographic method with the help of NIST and WILEY library as shown in Table-2. 


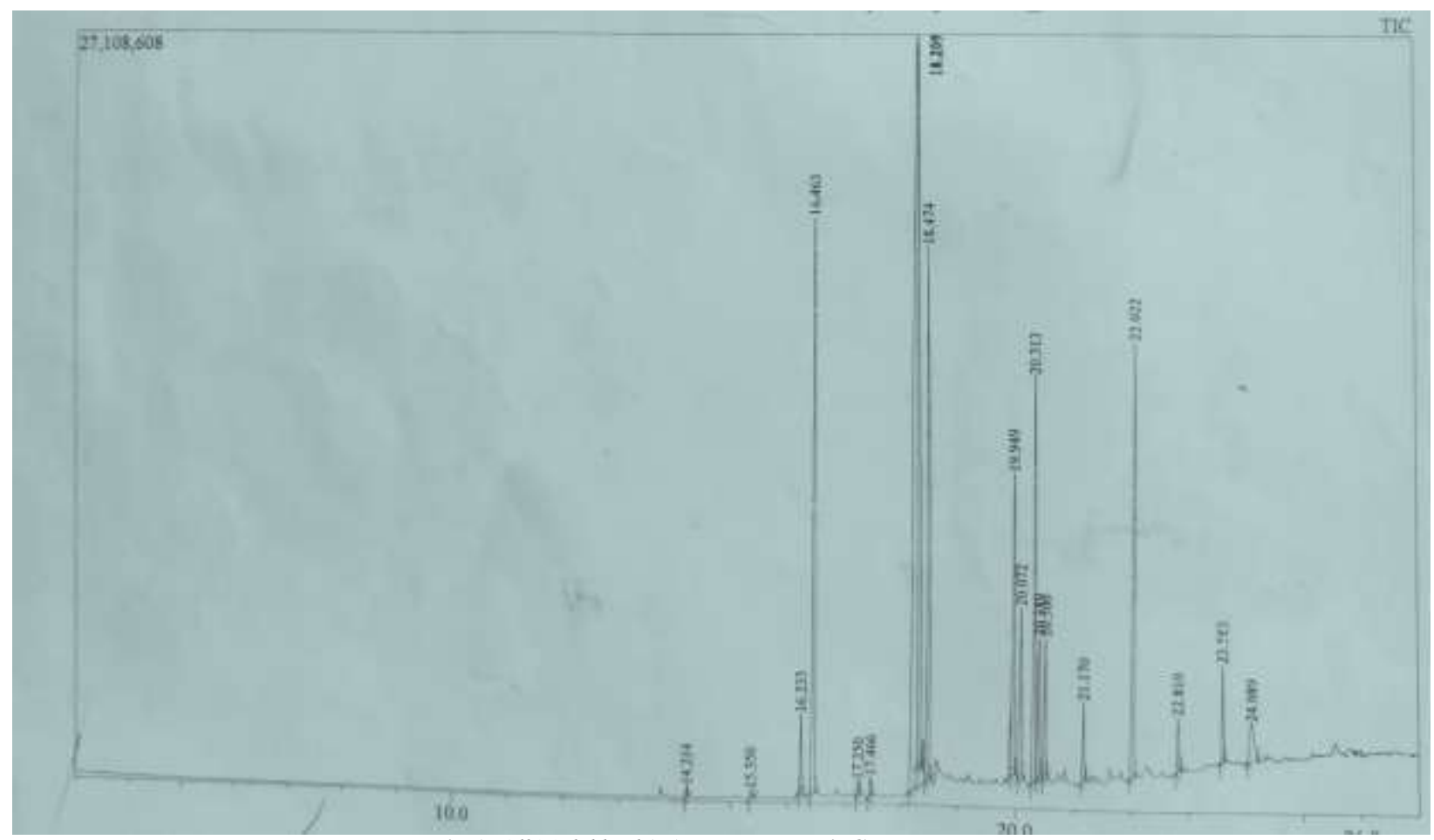

Fig-1: Albizialebbeck(L.) Benth seeds oil Chromatogram

Table-2: Fatty acids constituent of Albizialebbeck(L.) Benth seeds oil

\begin{tabular}{|l|l|l|l|}
\hline Peak No. & Name & R.Time & Area $\%$ \\
\hline 1 & Methyl tetradecanoate & 14.214 & 0.17 \\
\hline 2 & Pentadecanoic acid ,methyl ester & 15.350 & 0.12 \\
\hline 3 & 9-Hexadecenoic acid, methyl ester, $(Z)$ & 16.235 & 1.11 \\
\hline 4 & Hexadecenoic acid, methyl ester, $(\mathrm{Z})$. & 16.463 & 12.32 \\
\hline 5 & Cis-10- heptadecenoic acid, methyl ester & 17.250 & 0.22 \\
\hline 6 & heptadecenoic acid, methyl ester & 17.466 & 0.27 \\
\hline 7 & $9,12-$ Octadecadienoic acid (Z,Z),methyl ester & 18.250 & 29.09 \\
\hline 8 & 9-Octadecenoic acid (Z),methyl ester & 18.295 & 13.03 \\
\hline 9 & Methyl Stearate & 18.474 & 9.72 \\
\hline 10 & E,E,Z-1,3,12-Nonadecatriene-5,14-diol & 19.949 & 5.39 \\
\hline 11 & Oxaraneoctanoic acid,3-octyl,methyl ester & 20.017 & 3.58 \\
\hline 12 & Eicosenoic acid, methyl ester & 20.313 & 7.31 \\
\hline 13 & PGTI, methyl ester & 20.389 & 2.21 \\
\hline 14 & Mehtyl-13,16-Docosandienoate & 20.500 & 2.24 \\
\hline 15 & Heneicosanoic acid, methyl ester & 21.170 & 1.19 \\
\hline 16 & Docosenoic acid, methyl ester & 22.022 & 8.19 \\
\hline 17 & Tricosanoic acid, methyl ester & 22.810 & 0.88 \\
\hline 18 & Tetracosanoic acid, methyl ester & 23.583 & 1.44 \\
\hline 19 & Gamma.sitosterol & 24.89 & 1.53 \\
\hline Total of components & & $100 \%$ \\
\hline Total Saturated fatty acids & & \\
\hline Total Unsaturated fatty acids 82.90 & & \\
\hline- & Total of Mono unsaturated fatty acids 46.18 & \\
\hline & Total of Poly unsaturated fatty acids 36.72 & & \\
\hline
\end{tabular}

GC/MS analysis of Albizialebbeck(L.) Benth seeds oil provided a rich source of unsaturated fatty acids $(82.90 \%)$, important among which is linoleic acid with hypocholesterolemic effect, implying a cardioprotective property, then the amount of saturated fatty acids is $(17.10 \%)$, but unsaturated fatty acids contains monounsaturated fatty acids $(46.18 \%)$ and polyunsaturated fatty acids $(36.72 \%)$. The fatty acids acquire high percentage in Albizialebbeck(L.) Benth seeds oil were 9,12-Octadecadienoic acid (29.09\%), 9Octadecenoic acid (13.03\%), Hexadecenoic acid, methyl ester, (Z).(12.31), Methyl Stearate (9.72), Docosenoic acid, methyl ester $(8.19 \%)$, Eicosenoic acid, methyl ester (7.31), E,E,Z-1,3,12-Nonadecatriene- 
5,14-diol d (5.39\%), Oxaraneoctanoic acid, 3-octyl, methyl ester (3.58), other fatty acids which were a counted in percentage of the total fatty acids is modest amount.

\section{Antimicrobial Activity}

The oil was screened for antimicrobial activity against five standard microorganisms. The average of the diameters of the growth inhibition zones are shown in Table-3. The results were interpreted in terms of the commonly used terms (>9mm: inative; 9-12mm: partially active; $13-18 \mathrm{~mm}$ : active; $<18 \mathrm{~mm}$ :very active). Table-3 represented the antimicrobial activity of standard antibacterial and antifungal chemotherapeutic agents against standard bacteria and fungi respectively. The oil showed activity against Pseudomonas aeruginosa and partial activity against Escherichia coli, but it is inactive against Staphylococcus aureus, Bacillus subtilis, Staphylococcus auras and the fungi Candida albicans.

Table-3: Antimicrobial activity of standard drugs and Albizialebbeck(L.) Benth seeds oil.

\begin{tabular}{|l|l|l|l|l|l|l|}
\hline \multirow{2}{*}{ Name of compound } & \multirow{2}{*}{ Conc. } & \multicolumn{5}{|l|}{ Minimal inhibition concentration $\left(\boldsymbol{\mu g} \mathbf{~ m L}^{-\mathbf{1}}\right)$} \\
\cline { 3 - 7 } & & Gram-negative & Gram-positive & Fungal species \\
\cline { 3 - 7 } & & P.a & E.c. & B.s. & S.a & C.a \\
\hline Albizialebbeck(L.) Benth seeds oil & 100 & 15 & 11 & - & - & - \\
\hline Gentamycin & 40 & 21 & 22 & 25 & 19 & - \\
& 20 & 15 & 18 & 22 & 18 & - \\
& 10 & 12 & 15 & 17 & 14 & - \\
\hline Nystatin & - & - & - & - & - & 100 \\
\hline
\end{tabular}

\section{CONCLUSIONS}

In the present study, the authors reported the chemical constituents and biological activities of the Albizialebbeck(L.) Benth seeds oil. The different part of plant are showed Biological activities that is this plant display a multidisciplinary usage in treating several diseases. Further separation and identification of compound present in it may give new biologically active compounds, which can be used as lead compounds in future.

\section{ACKNOWLEDGEMENTS}

The authors we are very grateful to thank Dr. Mohammed Elhafez and all members of Department of Chemistry and Biology and the members of laboratory of chemistry, Faculty of Education, Omdurman Islamic University.

\section{REFERENCES}

1. Mishra SS, Gothecha VK, Sharma A. Albizia lebbeck: a short review. Journal of herbal medicine and toxicology. 2010;4(2):9-15.

2. Tasnim J, Saha A, Ahmed S, Sultana N, Muslim T, Rahman MA. Biological studies of the bark of Albizia Lebbeck (L.) Benth. International Journal of Pharmaceutical Sciences and Research. 2014 Nov 1;5(11):4969-4974.

3. Singh P, Agrawal T. Analysis of Fatty acids composition of the seed oil of the Albizia lebbeck (L.) Benth. Int. J. Sci. Res. in Multidisciplinary Studies Vol. 2018 Sep;4:9: 17-21.

4. Rudramani D, Parvesh K, Rajeev R. Shirish: description of a vishghana (antidote) dravya in
Ayurveda. World J of Pharmaceutical Research. 2018; 8(1):509-517.

5. Ambika K, Jagadeesan M. Comparative pharmacognostical studies on Albizia lebbeck (L.) wild and Albizia procera (Roxb.) Benth. leaves. International Journal of Innovative Research in Science, Engineering and Technology. 2017;6(9):19483-93.

6. Wati M, Khabiruddin M. Comparision of antioxidants in phenol extract and methanol extract of Albizia lebbeck from two locations. Int J Pharm Sci Rev Res Int. 2017;45(1):78-82.

7. Barmak A, Ezadi E, Hassanzadeh S, Adelian E, Poladi N, Hajeb A. Profile Comparison of Fatty Acids of Seeds of Albizia Lebbeck Trees, Native of Bushehr Province, with Some Common Oils. J. Appl. Environ. Biol. Sci. 2014 Jul 4;4(3s):31-4.

8. Chakravarthy VS, Negi PS. Enhanced in vitro regeneration from seedling explants of a medicinally important leguminous tree (Albizia lebbeck Benth.). Int. J. Curr. Microbiol. App. Sci. 2014;3(9):65-73.

9. Harmeet KA, Inderpal K. Shirish as an Antidote in Ayurveda. Int $\mathbf{J}$ Ayu Pharm Chem, Int J Ayu Pharm Chem. 2017; 7(2):64-67.

10. Uzma S, Zohaib R, Fareeha A, Bashir A, Sundas H, Tahir A. Experimental and Computational Studies to Characterize and Evaluate the Therapeutic E_ect of Albizialebbeck(L.) Seeds in Alzheimer's Disease Medicina, 2019; 55:184.

11. Adubiaro H, Olaofe O, Ogunbusola E, Adeyeye E. Nutritional and antinutritional attributes of underutilized tree crops: adansonia digitata, albizzia lebbeck and daniellia oliveri seeds. E. S. U. J. of Science and Technology (EJST). 2016; 2(1):24-33. 